EPJ Web of Conferences 36, 00016 (2012)

DOI: $10.1051 /$ epjconf/20123600016

C Owned by the authors, published by EDP Sciences, 2012

\title{
Hadron Medium Modifications
}

\author{
Steffen Strauch ${ }^{1, \mathrm{a}}$ \\ ${ }^{1}$ University of South Carolina, Columbia, SC 29208
}

\begin{abstract}
Proton recoil polarization in quasielastic electron scattering is a powerful tool to study properties of nucleons bound in the nuclear medium. The most precise results have been obtained at Jefferson Lab in the ${ }^{4} \mathrm{He}\left(\mathbf{e}, e^{\prime} \mathbf{p}\right)^{3} \mathrm{H}$ reaction at four momentum transfers of $Q^{2}=0.8(\mathrm{GeV} / c)^{2}$ and at $1.3(\mathrm{GeV} / c)^{2}$. The ratio of polarization-transfer components, $P_{x}^{\prime} / P_{z}^{\prime}$, is significantly reduced compared to the ratio obtained in elastic ${ }^{1} \mathrm{H}\left(\mathbf{e}, e^{\prime} \mathbf{p}\right)$ scattering. This depletion is well described with models which include in-medium modifications of the proton form factor or, alternatively, by strong charge-exchange final-state interactions. A future experiment will measure the dependence of this effect on the momentum of the struck nucleon in both, the ${ }^{4} \mathrm{He}\left(\mathbf{e}, e^{\prime} \mathbf{p}\right)^{3} \mathrm{H}$ and ${ }^{2} \mathrm{H}\left(\mathbf{e}, e^{\prime} \mathbf{p}\right) n$ reactions.
\end{abstract}

\section{Introduction}

Nucleon structure functions can be extracted from deep inelastic lepton scattering cross sections. These functions relate to the parton distributions of the nucleon. Cross section measurements of the European Muon Collaboration (EMC) on iron found a depletion of the deep inelastic structure function observed in the valence quark regime if compared to measurements on deuterium [1]. These findings have been confirmed by many measurements over a wide range of nuclei; see e.g. [2] for a review. In a recent experiment Seely et al. [3] measured the EMC effect for very light nuclei. While the specific causes of the modifications observed in the nuclear structure functions have not yet been identified with certainty, it is unlikely that the depletion can be understood in a conventional nucleon-meson treatment of nuclear physics [4]. In fact, a wide variety of QCD-based models, including quark-meson coupling [5,6] and chiral-quark soliton $[7,8]$ models, predict that the nucleons change properties with increasing nuclear density. These models do not only describe the EMC effect while being consistent with Drell-Yan data [7], they also predict observable changes in the electromagnetic form factors in nuclear matter. The predicted form-factor modifications are generally consistent with present constraints for both the electric form factor (from the Coulomb Sum Rule, for $\mathrm{Q}^{2}<0.5(\mathrm{GeV} / \mathrm{c})^{2}[9-11]$ ) and the magnetic form factor (from a $y$-scaling analysis [12] for $\left.\mathrm{Q}^{2}>1(\mathrm{GeV} / \mathrm{c})^{2}\right)$, and with limits on the scaling of nucleon magnetic moments in nuclei [13].

Measurements of the transverse and longitudinal polarization-transfer observables, $P_{x}^{\prime}$ and $P_{z}^{\prime}$, in the ${ }^{1} \mathrm{H}\left(\mathbf{e}, e^{\prime} \mathbf{p}\right)$ reaction have been extensively used to extract the ratio of the electric to magnetic Sachs form factors of the proton, $G_{E}^{p}$ and $G_{M}^{p}$, for the proton [14]. In the one-photon exchange approximation $G_{E}^{p} / G_{M}^{p}$ is directly proportional to the observable $P_{x}^{\prime} / P_{z}^{\prime}[15,16]$ :

$$
\frac{G_{E}^{p}}{G_{M}^{p}}=-\frac{P_{x}^{\prime}}{P_{z}^{\prime}} \cdot \frac{E+E^{\prime}}{2 m_{p}} \tan (\theta / 2),
$$

where $E$ and $E^{\prime}$ are the energies of the incident and scattered electron, $\theta$ is the electron scattering angle, and $m_{p}$ is the proton mass. The induced polarization $P_{y}$ in elastic ep scattering is zero in this approximation. Experimentally the proton polarization is determined from azimuthal asymmetries in

\footnotetext{
a e-mail: strauch@sc.edu
}

This is an Open Access article distributed under the terms of the Creative Commons Attribution License 2.0, which permits unrestricted use, distribution, and reproduction in any medium, provided the original work is properly cited. 


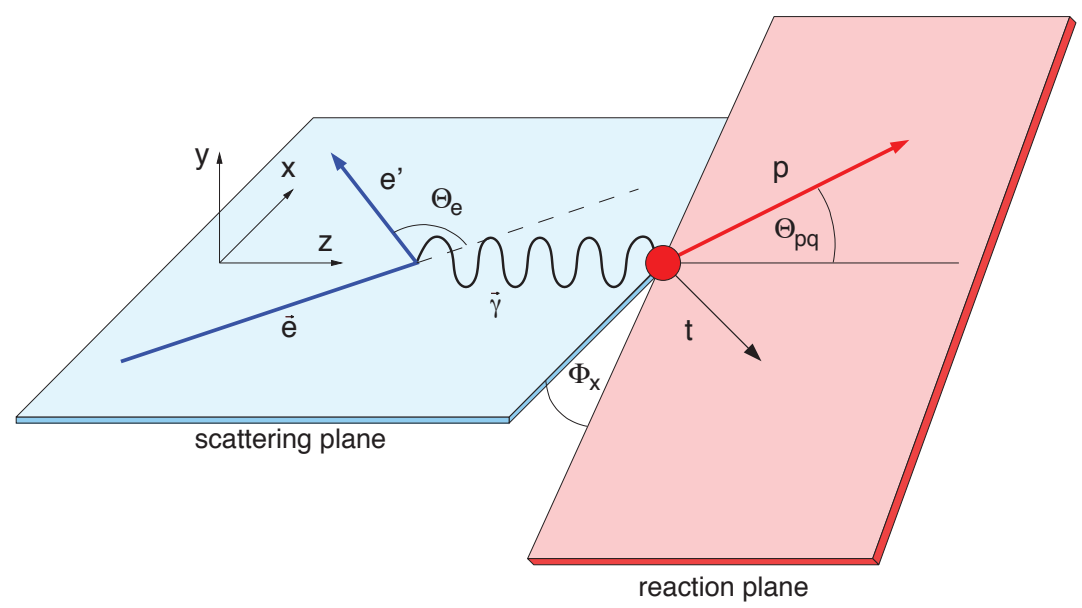

Fig. 1. Schematic of the ${ }^{4} \mathrm{He}\left(\mathbf{e}, e^{\prime} \mathbf{p}\right)^{3} \mathrm{H}$ reaction. The components of the proton recoil polarization are reconstructed in a coordinate system where the $z$ axis is along the three-momentum transfer, $\mathbf{q}$, the $y$ axis is perpendicular to the scattering plane, and the $x$ axis is in the scattering plane perpendicular to the momentum transfer, forming a right-handed system.

the angular distribution of the protons after scattering in the analyzer, typically carbon, of a focal plane polarimeter.

Measuring $P_{x}^{\prime} / P_{z}^{\prime}$ on a nuclear target in quasi-elastic proton knockout, $A\left(\mathbf{e}, e^{\prime} \mathbf{p}\right)$, is an intuitive method to investigate the properties of nucleons in the nuclear medium; see Fig. 1 for a definition of the reference system. The polarization-transfer double ratio

$$
R=\frac{\left(P_{x}^{\prime} / P_{z}^{\prime}\right)_{A}}{\left(P_{x}^{\prime} / P_{z}^{\prime}\right)_{1} \mathrm{H}}
$$

is then an experimental observable which directly reveals medium effects. It can be measured with small systematic uncertainties. To which extend these medium effects are due to changes in the wave function of the bound nucleons or due to more conventional many-body effects, such as mesonexchange currents (MEC), isobar configurations (IC), and final-state interactions (FSI), can only be determined within the context of a model. In that context polarization-transfer observables provide us with a way to study the behavior of the nucleon form factors in the nuclear medium. Excellent control over the reaction mechanism effects is certainly required [17]. At high momentum transfer, however, the contribution of many-body and rescattering mechanisms are strongly suppressed [18].

An important constraint of these rescattering mechanisms comes from the induced polarization $P_{y}$ of the ejected proton. In the one-photon exchange approximation the induced polarization in quasielastic electron scattering is zero when no FSI between the ejected nucleon and the residual nuclear system are considered [19]. Induced-polarization data can be taken simultaneously to polarization-transfer data, however, they are often plagued by sizable systematic uncertainties due to possible instrumental asymmetries.

\section{Present Experimental Results}

Polarization transfer in the quasielastic $A\left(\mathbf{e}, e^{\prime} \mathbf{p}\right)$ reaction has been measured on ${ }^{2} \mathrm{H}[20-23]$, on ${ }^{4} \mathrm{He}$ [24-27], and, with rather large statistical uncertainties, on ${ }^{16} \mathrm{O}$ [28]. In these exclusive experiments a highly polarized electron beam was incident on the target and the scattered electron and ejected proton were detected in coincidence in two high-resolution spectrometer arms. The missing-mass technique was used to identify the residual nucleus in the final state. The proton spectrometer was equipped with a focal plane polarimeter, which measures the asymmetry of polarized protons scattered from a carbon 


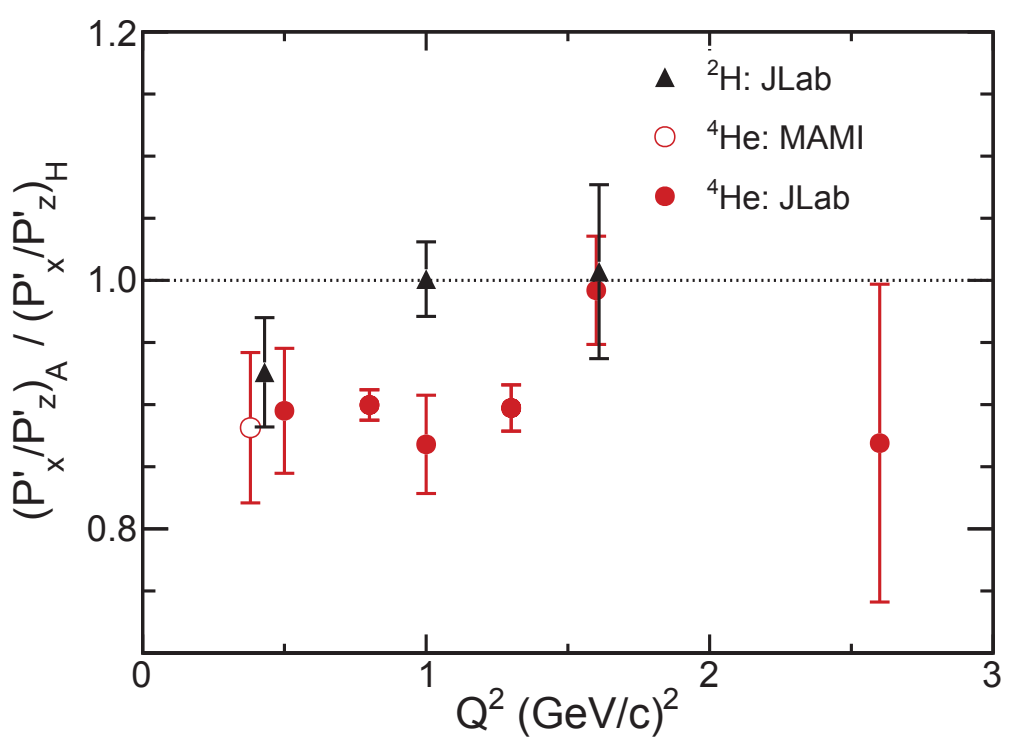

Fig. 2. Polarization-transfer ratio, $\left(P_{x}^{\prime} / P_{z}^{\prime}\right)_{A}$, for ${ }^{2} \mathrm{H}\left(\mathbf{e}, e^{\prime} \mathbf{p}\right) n$ (triangles) from [23] and for ${ }^{4} \mathrm{He}\left(\mathbf{e}, e^{\prime} \mathbf{p}\right)^{3} \mathrm{H}(\mathrm{circles})$ from [24-26] normalized to the polarization-transfer ratio for ${ }^{1} \mathrm{H}\left(\mathbf{e}, e^{\prime} \mathbf{p}\right)$. All data were taken at low missing momentum.

analyzer [29]. Figure 2 compares the polarization-transfer double ratio from ${ }^{2} \mathrm{H}$ and ${ }^{4} \mathrm{He}$ for various values of the four-momentum transfer, $Q^{2}$. All measurements were taken at low missing momentum to minimize conventional many-body effects. Within statistical uncertainties, no evidence of medium modifications are found in ${ }^{2} \mathrm{H}$. This is not surprising as the sampled density in ${ }^{2} \mathrm{H}$ is small and the nucleons are only weakly bound. The mean value of the polarization-transfer double ratio from the much denser ${ }^{4} \mathrm{He}$, however, is significantly different from one. Also the per-nucleon deep inelastic structure function in ${ }^{4} \mathrm{He}$ is significantly different than that for deuterium [3]. While the ${ }^{4} \mathrm{He}$ doubleratio at $Q^{2}=1.6(\mathrm{GeV} / c)^{2}$ is consistent with one, a statistical analysis seems to indicate that this could merely be a statistical fluctuation.

Figure 3 shows the ${ }^{4} \mathrm{He}\left(\mathbf{e}, e^{\prime} \mathbf{p}\right)^{3} \mathrm{H}$ polarization-transfer double ratio (upper panel) and the induced polarization, $P_{y}$, (lower panel) from JLab experiments E93-049 [25] and E03-104 [26,27]. The $P_{y}$ data have been corrected for the spectrometer acceptance and its comparison with models is made for missing momentum $p_{m} \approx 0$. Dedicated calibration data were taken during experiment E03-104 at $Q^{2}=0.8$ and $1.3(\mathrm{GeV} / \mathrm{c})^{2}$ to significantly reduce the otherwise sizable systematic uncertainties in the extraction of $P_{y}$ [27]. The data are compared with results of a relativistic distorted-wave impulse approximation (RDWIA) calculation by the Madrid group [31-33]. In its standard form the calculation uses the free nucleon form factors in the current operator of the calculation (dotted curves). Results are shown for two different choices of the current operator: $c c 1$ and $c c 2$ as defined in [35]. FSI are taken into account using an updated version of the RLF relativistic optical potentials [36,37]. No charge-exchange FSI were included. Preliminary studies showed that their effect is small [38]. The good description of the induced polarization lends support to the modeling of FSI in this model with the $c c l$ operator. The polarization-transfer ratio, however, is too large and does not describe the data. The solid and dashed curves show the results of the Madrid model after replacing the free nucleon form factors with density-dependent medium-modified form factors from the QMC [5] and CQS [8] models in the current operator. The inclusion of in-medium form factors strongly changes the result for the polarization-transfer ratio, as expected from Eq. (1), and brings the calculation in very good agreement with the data. At the same time it leaves the induced polarization rather unaffected. 

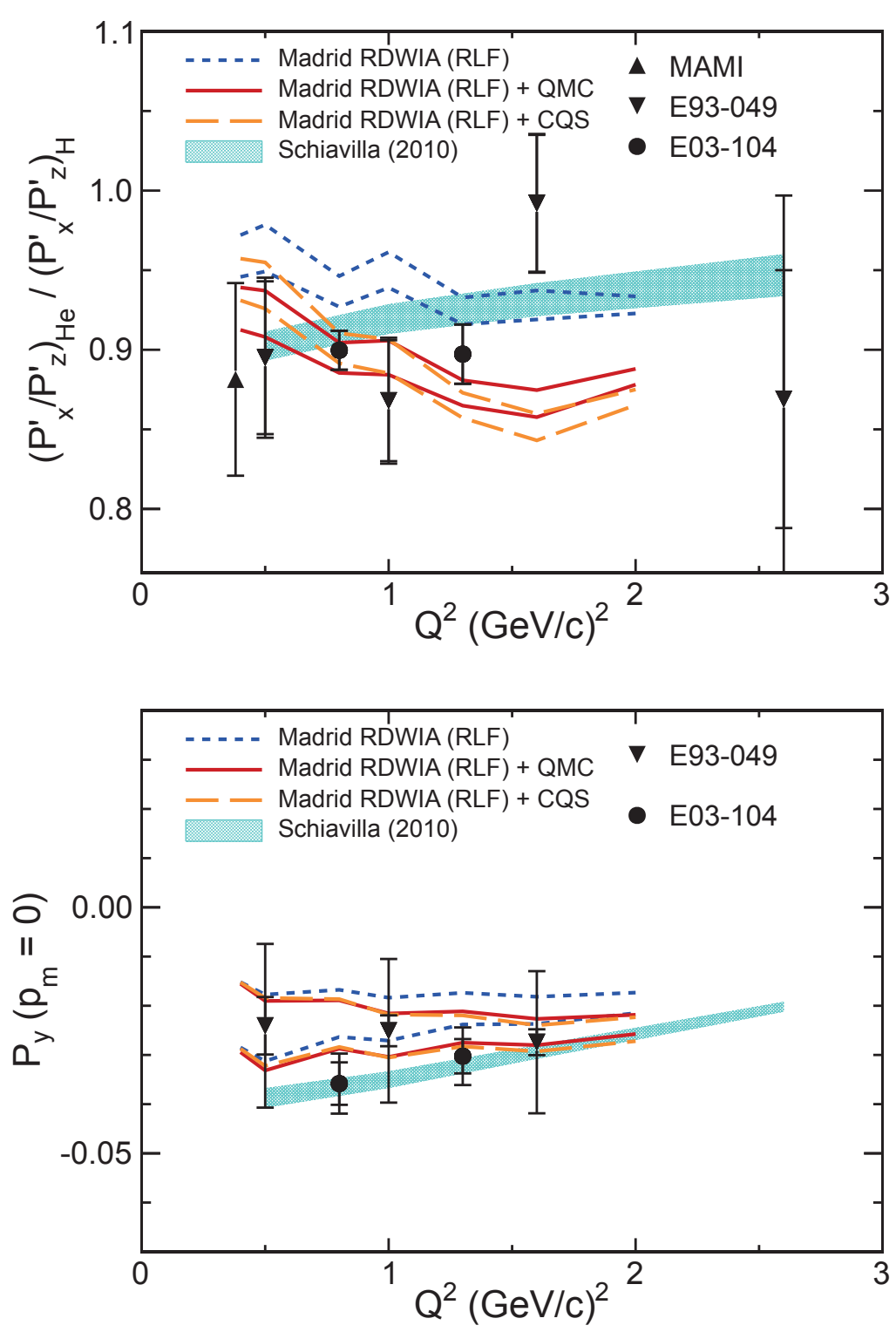

Fig. 3. ${ }^{4} \mathrm{He}\left(\mathbf{e}, e^{\prime} \mathbf{p}\right)^{3} \mathrm{H}$ polarization-transfer double ratio $R$ (upper panel) and induced polarization $P_{y}$ (lower panel) as a function of $Q^{2}$ from MAMI, Mainz [24] and Jefferson Lab E93-049 [25] (open symbols) and E03-104 [26, 27] (filled circles) experiments. The data are compared to calculations from Schiavilla et al. [30] and the Madrid group [31-33] using the $c c 1$ (lower set of curves) and $c c 2$ (upper set of curves) current operators. In-medium form factors from the QMC [5] (solid curve) and CQS [8] (dashed curve) models were used in two of the Madrid calculations. Note that the $P_{y}$ data have been corrected for the spectrometer acceptance and the comparison with models is made for missing momentum $p_{m} \approx 0$. Figure taken from [34].

Very different calculations are provided by Schiavilla et al. [30] and are shown in Fig. 3 as shaded bands. These calculations also give a good description of the data. That model uses variational wave functions for the bound three- and four-nucleon systems, non-relativistic MEC, and free nucleon form factors. The FSI are treated within the optical potential framework and include both spin-independent 
and spin-dependent charge-exchange terms. Parameters of the FSI model have been constrained by the new $P_{y}$ data from E03-104.

\section{Possible Future Experiments}

The existing ${ }^{4} \mathrm{He}\left(\mathbf{e}, e^{\prime} \mathbf{p}\right)^{3} \mathrm{H}$ polarization-transfer data can be well described by either the inclusion of medium-modified form factors or strong charge-exchange FSI in the models. While the Madrid and Schiavilla et al. models are quite different, their free-form-factor results do agree with each other for $Q^{2} \geq 1.3(\mathrm{GeV} / c)^{2}$ where ambiguities in the choice of the current operator become smaller. The effect of in-medium modifications of the nucleon form factors, however, reduces the polarization-transfer double ratio by at least 5\%, as shown by the Madrid calculations in Fig. 3. An effect that large is easily observable. After the energy upgrade of Jefferson Lab, experiment E12-11-002 [39] will measure with high precision the proton recoil polarization at $Q^{2}=1.8(\mathrm{GeV} / c)^{2}$. Compared to the before mentioned measurements this experiment will benefit from the larger spectrometer acceptances in JLab Hall C and a substantial increase in beam time; for technical details see [39].

The present studies in the ${ }^{4} \mathrm{He}\left(\mathbf{e}, e^{\prime} \mathbf{p}\right)^{3} \mathrm{H}$ reaction were performed at small missing momentum to minimize conventional many-body effects and final-state interactions. This is a reasonable approach if medium modifications depend only on the mean nuclear density. If, as Ciofi degli Atti et al. [40] argue, the modification of the bound-nucleon wave function depends on the momentum of the nucleon the approach minimizes observable effects. In fact, Weinstein et al. [41] found that the magnitude of the EMC effect is linearly related to the per-nucleon probabilities of nucleon-nucleon short-range correlations (SRC). SRCs occur between pairs of nucleons with high relative momentum and low center of mass momentum. The observed linearity thus supports the idea that the EMC effect is caused by high-momentum nucleons in the nucleus. A second part of E12-11-002 will therefore be a detailed study of the polarization-transfer observables as a function of missing momentum.

In the impulse approximation the missing momentum can be related to the momentum, $p$, of the stuck nucleon through $p^{2}=\left(m_{A}-E_{m}\right)^{2}-p_{m}^{2}$, where $E_{m}$ and $p_{m}$ are respectively the missing energy and momentum in the $A\left(e, e^{\prime} p\right)$ reaction. The proton virtuality is then defined as $v=p^{2}-m_{p}^{2}$. Figure 4 shows the polarization-transfer double ratio for the present and for the expected data as a function of the proton virtuality. Explicit results for the electromagnetic form factors as a function of the momentum of the off-shell proton have been obtained by Kondratyuk et al. [42] with a dressed $K$-matrix model. E12-11-002 will measure both the ${ }^{4} \mathrm{He}\left(\mathbf{e}, e^{\prime} \mathbf{p}\right)^{3} \mathrm{H}$ and ${ }^{2} \mathrm{H}\left(\mathbf{e}, e^{\prime} \mathbf{p}\right) n$ reactions at $Q^{2}=1.0(\mathrm{GeV} / c)^{2}$ over a wide range of proton virtualities. While the average densities of ${ }^{2} \mathrm{H}$ and ${ }^{4} \mathrm{He}$ are very different, the experiment can probe similar proton virtualities in both reactions at larger missing momenta and is thus uniquely suited to study the density and momentum dependence of the bound nucleon knockout.

The experiments discussed so far only probe medium effects in the quasielastic $\left(\mathbf{e}, e^{\prime} \mathbf{p}\right)$ reaction. Complementary measurements in the $\left(\mathbf{e}, e^{\prime} \mathbf{n}\right)$ could provide important new insides. Studies found a marked difference of the in-medium modification of the nucleon electromagnetic form factor ratio for the proton and for the neutron at low $Q^{2}$ [43]. The in-medium ratio of the neutron $G_{E}^{n} / G_{M}^{n}$ form factor ratio is predicted to increase relative to the free result, while the ratio for the proton is expected to decrease in the medium. A comparison of high-precision measurements of the reactions ${ }^{2} \mathrm{H}\left(\mathbf{e}, e^{\prime} \mathbf{n}\right) p$ and ${ }^{4} \mathrm{He}\left(\mathbf{e}, e^{\prime} \mathbf{n}\right)^{3} \mathrm{He}$ would allow to test these predictions.

\section{Summary}

The ratio of polarization-transfer coefficients, $P_{x}^{\prime} / P_{z}^{\prime}$, in the quasi-elastic nucleon-knockout reaction is arguably one of the most direct experimental observables to identify nuclear-medium changes to nucleon properties as other conventional medium effects are suppressed. Furthermore, the possible role of FSI in the interpretation of these data can be constrained by the induced polarization $P_{y}$. After such constraints, present ${ }^{4} \mathrm{He}\left(\mathbf{e}, e^{\prime} \mathbf{p}\right)^{3} \mathrm{H}$ polarization-transfer data can be well described by either the inclusion of medium-modified form factors or strong charge-exchange FSI in the models. 


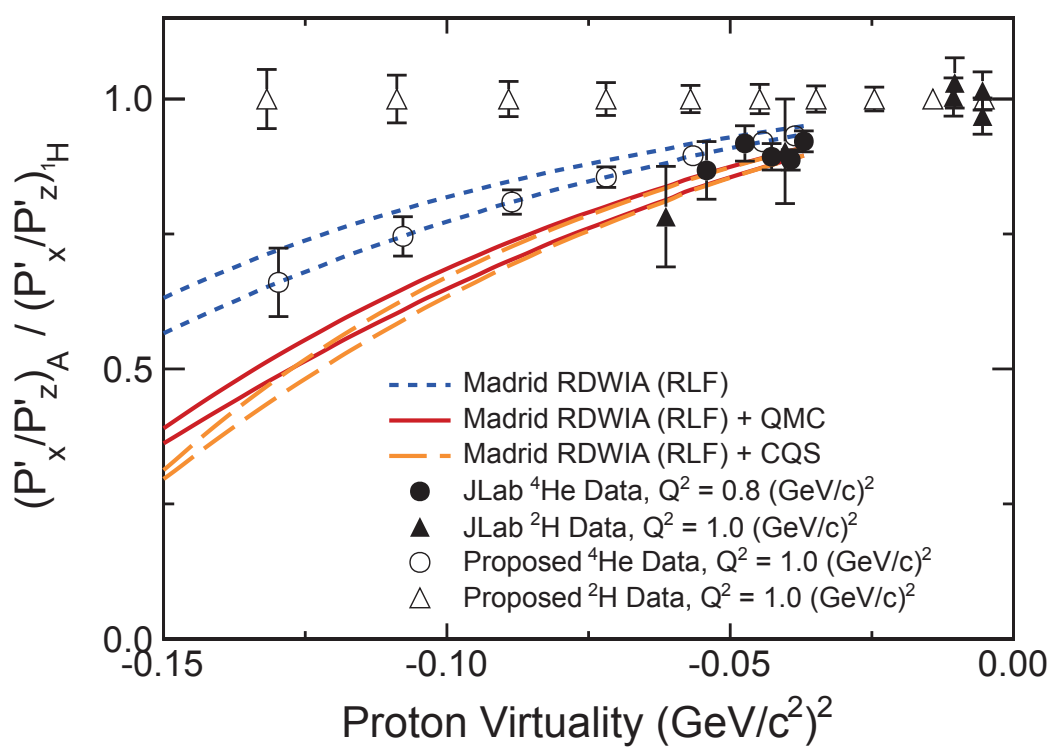

Fig. 4. Polarization-transfer double ratio in the ${ }^{4} \mathrm{He}\left(\mathbf{e}, e^{\prime} \mathbf{p}\right)^{3} \mathrm{H}$ and ${ }^{2} \mathrm{H}\left(\mathbf{e}, e^{\prime} \mathbf{p}\right) n$ reactions The curves are various calculations using the Madrid RDWIA model for the reaction on ${ }^{4} \mathrm{He}$ and current operators $c c 1$ (lower set of curves) and $c c 2$ (upper set of curves). The points indicate existing data [23,26] (solid symbols) and the statistical uncertainties of the expected data from E12-11-002 [39] which are arbitrarily placed on the RDWIA ( $c c 1)$ curve for ${ }^{4} \mathrm{He}$ and at $R=1$ for ${ }^{2} \mathrm{H}$.

An upcoming measurement of the quasielastic $\left(\mathbf{e}, e^{\prime} \mathbf{p}\right)$ reaction off both ${ }^{4} \mathrm{He}$ and ${ }^{2} \mathrm{H}$ targets will allow to further probe the momentum dependence of the bound nucleon electromagnetic current, including possible medium modifications of the proton electromagnetic form factor.

\section{Acknowledgments}

This work was supported in parts by the U.S. National Science Foundation: NSF PHY-0856010. Jefferson Science Associates operates the Thomas Jefferson National Accelerator Facility under DOE contract DE-AC05-06OR23177.

\section{References}

1. J. Aubert et al., Phys.Lett. B123 (1983) 275.

2. P. Norton, Rept.Prog.Phys. 66 (2003) 1253.

3. J. Seely et al., Phys. Rev. Lett. 103 (2009) 202301.

4. G. A. Miller and J. R. Smith, Phys. Rev. C65 (2002) 015211.

5. D.-H. Lu, A. W. Thomas, K. Tsushima, A. G. Williams, and K. Saito, Phys. Lett. B417 (1998) 217.

6. D.-H. Lu, K. Tsushima, A. W. Thomas, A. G. Williams, and K. Saito, Phys. Rev. C60 (1999) 068201.

7. J. R. Smith and G. A. Miller, Phys.Rev.Lett. 91 (2003) 212301.

8. J. R. Smith and G. A. Miller, Phys. Rev. C70 (2004) 065205.

9. J. Jourdan, Phys. Lett. B353 (1995) 189.

10. J. Morgenstern and Z. E. Meziani, Phys. Lett. B515 (2001) 269.

11. J. Carlson, J. Jourdan, R. Schiavilla, and I. Sick, Phys. Lett. B553 (2003) 191. 
12. I. Sick, Comments Nucl. Part. Phys. A18 (1988) 109.

13. T. E. O. Ericson and A. Richter, Phys. Lett. B183 (1987) 249.

14. C. Perdrisat, V. Punjabi, and M. Vanderhaeghen, Prog.Part.Nucl.Phys. 59 (2007) 694.

15. A. I. Akhiezer and M. P. Rekalo, Sov. J. Part. Nucl. 4 (1974) 277.

16. R. G. Arnold, C. E. Carlson, and F. Gross, Phys. Rev. C23 (1981) 363.

17. J. W. Van Orden, Phys. Rev. C74 (2006) 034607.

18. J.-M. Laget, Nucl. Phys. A579 (1994) 333.

19. A. Picklesimer and J. Van Orden, Phys.Rev. C35 (1987) 266.

20. D. Eyl et al., Z. Phys. A352 (1995) 211.

21. B. D. Milbrath et al., Phys. Rev. Lett. 80 (1998) 452.

22. D. H. Barkhuff et al., Phys. Lett. B470 (1999) 39.

23. B. Hu et al., Phys. Rev. C73 (2006) 064004.

24. S. Dieterich et al., Phys. Lett. B500 (2001) 47.

25. S. Strauch et al., Phys. Rev. Lett. 91 (2003) 052301.

26. M. Paolone et al., Phys. Rev. Lett. 105 (2010) 072001.

27. S. Malace, M. Paolone, S. Strauch, I. Albayrak, J. Arrington, et al., Phys.Rev.Lett. 106 (2011) 052501 .

28. S. Malov et al., Phys. Rev. C62 (2000) 057302.

29. V. Punjabi et al., Phys. Rev. C71 (2005) 055202.

30. R. Schiavilla, O. Benhar, A. Kievsky, L. E. Marcucci, and M. Viviani, Phys. Rev. Lett. 94 (2005) 072303.

31. J. M. Udias, J. A. Caballero, E. Moya de Guerra, J. E. Amaro, and T. W. Donnelly, Phys. Rev. Lett. 83 (1999) 5451.

32. J. A. Caballero, T. W. Donnelly, E. Moya de Guerra, and J. M. Udias, Nucl. Phys. A632 (1998) 323.

33. J. M. Udias and J. R. Vignote, Phys. Rev. C62 (2000) 034302.

34. S. Strauch, S. Malace, and M. Paolone, Fizika B 20 (2011) 149.

35. T. De Forest, Nucl. Phys. A392 (1983) 232.

36. C. J. Horowitz, Phys. Rev. C31 (1985) 1340.

37. D. P. Murdock and C. J. Horowitz, Phys. Rev. C35 (1987) 1442.

38. J.M. Udias, private communication (2010).

39. Jefferson Lab Experiment E12-11-002, "Proton Recoil Polarization in the ${ }^{1} \mathrm{H}\left(e, e^{\prime} p\right),{ }^{2} \mathrm{He}\left(e, e^{\prime} p\right) n$, and ${ }^{4} \mathrm{He}\left(e, e^{\prime} p\right)^{3} \mathrm{H}$ Reactions", E. Brash, G.M. Huber, R. Ransome, and S. Strauch, spokespersons.

40. C. Ciofi degli Atti, L. L. Frankfurt, L. P. Kaptari, and M. I. Strikman, Phys. Rev. C76 (2007) 055206.

41. L. Weinstein, E. Piasetzky, D. Higinbotham, J. Gomez, O. Hen, et al., Phys.Rev.Lett. 106 (2011) 052301 .

42. S. Kondratyuk, K. Kubodera, and F. Myhrer, Phys.Rev. C71 (2005) 028201.

43. I. C. Cloet, G. A. Miller, E. Piasetzky, and G. Ron, Phys. Rev. Lett. 103 (2009) 082301. 